\title{
Can foot placement during gait be trained? Adaptations in stability control when ankle moments are constrained
}

\author{
L.A. Hoogstad ${ }^{1 *}$, A.M. van Leeuwen ${ }^{1,2^{*}}$, J.H. van Dieën ${ }^{1,2}$, S.M. Bruijn ${ }^{1,2}$ \\ ${ }^{1}$ Department of Human Movement Sciences, Faculty of Behavioural and Movement Sciences, \\ Vrije Universiteit Amsterdam, Amsterdam Movement Sciences, Amsterdam, The Netherlands \\ ${ }^{2}$ Institute of Brain and Behavior Amsterdam \\ Email: a.m.van.leeuwen@vu.nl \\ *Shared first authorship
}

Accurate coordination of mediolateral foot placement, relative to the center of mass kinematic state, is one of the mechanisms which ensures mediolateral stability during human walking. Previously, we found that shoes constraining ankle moments decreased foot placement accuracy, presumably by impairing control over movement of the swing leg. As such, ankle moment constraints can be seen as a perturbation of foot placement. Direct mechanical perturbations of the swing leg trajectory can improve foot placement accuracy as an after-effect. Here, we asked whether constrained ankle moments could have a similar effect. If confirmed, this would offer a simple training tool for individuals with impaired foot placement control. Participants $(\mathrm{n}=19)$ walked in three conditions; normal (baseline, 10 minutes), while wearing shoes constraining ankle moments (training, 15 minutes), and normal again (after-effects, 10 minutes). Foot placement accuracy was calculated as the percentage of variance in foot placement that could be predicted based on the center of mass kinematic state in the preceding swing phase. When walking with constrained ankle moments, foot placement accuracy decreased initially compared to baseline, but it gradually improved over time. In the after-effect condition, foot placement accuracy was higher than during baseline, but this difference was not significant. When walking with constrained ankle moments, we observed increased step width, decreased stride time and reduced local dynamic stability. In conclusion, constraining ankle moment control deteriorates foot placement accuracy. A nonsignificant trend towards improved foot placement accuracy after prolonged exposure to constrained ankle moments, allows for speculation on a training potential. 


\section{Introduction}

Stable gait is crucial for activities of daily living. With aging, changes occur that affect the ability to maintain stability (Rubenstein, 2006; Vandervoort, 2002) and this constitutes a public health concern. Falls are common in older adults and can have severe impacts on their functionality and well-being (Rubenstein and Josephson, 2006). Furthermore, injuries related to falls lead to accumulating healthcare costs (Florence et al., 2018; Polinder et al., 2016).

Gait stability requires the coordination of the center of mass relative to the base of support (Bruijn and van Dieën, 2018). Different strategies can be used to establish appropriate coordination between the center of mass and the base of support (Reimann et al., 2018). Accurate foot placement relative to the body's kinematic state, seems to be the dominant strategy (Bruijn and van Dieën, 2018). Foot placement defines the borders of the base of support, and through foot placement the base of support can be enlarged or displaced to accommodate the ongoing movement of the center of mass (Hof, 2007; Reimann et al., 2018).

Previous work showed that foot placement can be predicted by the full body center of mass kinematic state during the preceding swing phase (Hurt et al., 2010; Mahaki et al., 2019; van Leeuwen et al., 2020; Wang and Srinivasan, 2014). The quality of foot placement control can thus be quantified as the predictability of foot placement based on center of mass kinematic state or proxies thereof.

This quality of foot placement control appears to be important to maintain stability. Following a stroke, individuals demonstrate less accurate foot placement control and less stable gait (Dean and Kautz, 2015). Similarly, older adults show less accurate foot placement than young adults (Arvin et al., 2018; Hurt et al., 2010), which may contribute to their elevated fall risk. Therefore, training interventions targeting foot placement control may help to improve gait stability. Recent research showed that in young adults, mechanical perturbations of foot placement caused improved foot placement accuracy as an after-effect (Heitkamp et al., 2019; Reimold et al., 2019).

In previous work, we found that walking with constrained ankle moments, wearing a customized shoe (LesSchuh), decreased foot placement accuracy (van Leeuwen et al., 2020). The LesSchuh is a shoe on which it is almost impossible to use mediolateral ankle moment control, due to a narrow ridge attached to the shoe's sole. The decrease in foot placement accuracy with constrained ankle moments may be caused by an inability to use stance leg ankle moments to control the subsequent foot placement. During targeted stepping, stance leg ankle moments contribute to accurate foot placement (Zhang et al., 2020), and also during normal steady-state walking a relationship exists between ankle moment control and subsequent foot placement (Fettrow et al., 2019). Constrained stance leg ankle moments can thus be seen as perturbations of foot placement of the contralateral leg. Considering the evidence for aftereffects of direct mechanical perturbations of the swing leg to alter foot placement (Reimold et al., 2019), prolonged exposure to ankle moment constraints may lead to improved foot placement accuracy in normal walking.

The purpose of the present study was to investigate whether foot placement becomes more accurate during and after walking with ankle moment constraints. To this end, participants walked for one training session on the LesSchuh.

We hypothesized that the ankle moment constraint imposed by the LesSchuh would cause an immediate negative effect on foot placement accuracy (H1), followed by an increase in foot placement accuracy throughout the training condition (H2). Furthermore, we hypothesized a positive after-effect when participants return to walking on their normal shoes (H3). Finally, we hypothesized that with time the after-effect would decrease (H4). In addition to changes in foot placement accuracy, we explored modulations in step width and stride frequency, as compensatory stabilizing mechanisms (van Leeuwen et al., 2020). In addition, to assess whether gait changes due to LesSchuh training affected gait stability, we calculated local divergence exponents (Bruijn et al., 2013). 


\section{Methods}

\subsection{Participants}

Participants were included if aged between 18 and 65 years. Participants with a self-reported history of neurological or other disorders that could affect the ability to walk independently were excluded. After explaining the experiment, participants signed written informed consent. The experimental design and procedures were approved by the Ethical committee (VCWE-2019-108).

\subsection{Experimental setup.}

During the experiment, two different shoes were used for each participant. The first shoe type was a normal shoe imposing no constraints on gait. The second type was a shoe which constrained ankle moments; the LesSchuh. A flexible ridge along the sole, as a limited base of support, constrained ankle moment control in the frontal plane (Figure 1). Due to the flexible ridge, normal roll-off and push-off can be accomplished, but mediolateral center of pressure shifts are constrained.

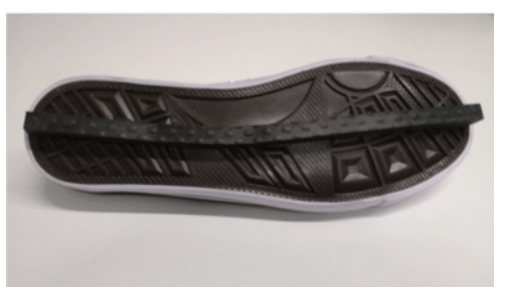

Figure 1. LesSchuh

Participants walked on a split-belt treadmill with a safety bar on either side. Full body kinematic data were recorded with Optotrak (Northern Digital Inc, Waterloo Ont, Canada) at a sampling rate of 50 samples/second. Cluster markers of three LEDs where placed on the feet, shank, thighs, pelvis, trunk, upper arms, and forearms. 36 anatomical landmarks were digitized by using a six-marker probe.

\subsection{Experimental design}

After placing the markers, the anatomical landmarks were digitized. Subsequently, participants were asked to walk on a split-belt treadmill in three conditions. During all three conditions the participant walked at constant $(1.25 *$ sqrt (leg length) $\mathrm{m} / \mathrm{s}$ ) walking speed. In the Baseline condition, the participant walked for 10 minutes with normal shoes. After this condition, the split-belt was stopped and the participant was asked to change shoes. In preparation of the 15-minute Training condition, the anatomical landmarks of both feet were digitized again, while the participant was wearing the LesSchuh. Then, participants were instructed to walk on the ridge of the shoe while trying to not touch the ground with the sides of the shoe's sole. In addition, participants were instructed to keep pointing their feet straight ahead, to avoid a toeing-out strategy (Rebula et al., 2017) as a compensation for the constrained ankle moments. After the Training condition, the split-belt was stopped and the participant was asked to change back to normal shoes and the anatomical landmarks were digitized again. In the After-effect condition, the participant walked for 10 minutes with normal shoes.

Table 1.

Overview of the performed conditions

\begin{tabular}{lll}
\hline Condition & Time & Type of shoe \\
\hline Baseline & 10 minutes & Normal shoes \\
Training & 15 minutes & LesSchuh \\
After-effect & 10 minutes & Normal shoes \\
\hline
\end{tabular}

\subsection{Data processing}

Data were processed in Matlab 2021A (The MathWorks Inc., Natick, MA). In case of missing marker coordinates due to limited visibility, data were interpolated using spline interpolation. Gait events (heel strike and toe-off) were detected from the "butterfly pattern" of the center of pressure derived from the force plate data (Roerdink et al., 2008). A step was defined as the period between toe-off and heel strike. 
Mid-swing was defined at 50 percent of the step. Step width (i.e. mediolateral foot placement) was calculated based on the position of the digitized heel markers at midstance. For estimation of the center of mass, the body was segmented in 16 segments: pelvis, abdomen, thorax, head, thighs, feet, upper arms, forearms and hands. For each segment, an estimation of the mass was made based on the segment circumference and length by using a regression equation (De Leva, 1996; Zatsiorsky, 1990). The total body center of mass was derived from a weighted sum of the body segments' center of masses.

We considered two epochs of 30 strides (the first 30 and last 30 strides of each condition) for statistical analysis. To investigate foot placement accuracy, linear regression was used to predict the following foot placement based on center of mass position and velocity during the preceding swing phase (Mahaki et al., 2019; van Leeuwen et al., 2020; Wang and Srinivasan, 2014). The ratio between the predicted foot placement variance and the actual foot placement variance was expressed as the relative explained variance $\left(\mathrm{R}^{2}\right)$ and was the main outcome in this study. A high relative explained variance indicates a stronger relation between center of mass state and the foot placement, i.e. higher foot placement accuracy. We used regression equation [1] (Bruijn, 2020) in which the mediolateral center of mass position $\left(\mathrm{CoM}_{\mathrm{pos}}\right)$ and velocity $\left(\mathrm{CoM}_{\mathrm{vel}}\right)$ at terminal swing predict mediolateral foot placement (FP). $\beta_{\mathrm{pos}}$ and $\beta_{\mathrm{vel}}$ represent the regression coefficients and $\varepsilon$ the residual of the model (i.e. the discrepancy between predicted and FP). For each epoch of 30 strides, we determined the $\mathrm{R}^{2}$ of this regression.

$$
\mathrm{FP}=\beta_{\mathrm{pos}} \cdot \mathrm{CoM}_{\mathrm{pos}}+\beta_{\mathrm{vel}} \cdot \mathrm{CoM}_{\mathrm{vel}}+\varepsilon,
$$

As a measure of local dynamic stability, local divergence exponents were determined (Bruijn et al., 2013; Bruijn, 2021; Mehdizadeh, 2019; Rosenstein et al., 1993) by first constructing a six-dimensional state space based on time-delayed ( 25 samples) copies of the 3D center of mass velocity. To this end, the signal was resampled so that on average each stride was 100 samples in length. Subsequently, we tracked the divergence for each time point and its five nearest neighbors for 1000 samples (equivalent to approximately ten strides). A nearest neighbor was defined as a point at the smallest Euclidian distance, at least half an average stride before or half an average stride after the current time point. We computed the average logarithmic divergence curve and fitted a line (least squares fit) to this curve over the first 50 samples (equivalent to approximately half a stride). Finally, we defined the local divergence exponent as the slope of this line. The higher the local divergence exponent, the less stable the gait pattern.

Step width was defined as the mean distance between mediolateral foot placements. Stride time was defined as the time between two heel strikes of the same leg.

The data and code for the analysis can be found online: https://surfdrive.surf.nl/files/index.php/s/ZgLVUg7ftfPkNTO , and will be uploaded to Zenodo after the paper has been accepted for publication.

\subsection{Statistical analysis}

To test for changes during the baseline trial, we performed paired t-tests between the start and end values of the baseline trial for the $\mathrm{R}^{2}$, stride time, step width and local divergence exponent.

To test each of our hypotheses we performed paired t-tests on the $\mathrm{R}^{2}$. For the training and after-effect conditions, the first and last 30 strides were used, representative of respectively the start and the end of each trial. To exclude any habituation to treadmill walking from this analysis, only the last 30 strides were included for the baseline condition. H1 stated that an ankle moment constraint would cause an immediate negative effect on foot placement accuracy. We thus expected a lower $\mathrm{R}^{2}$ at the start of the training as compared to the end of the baseline trial. $\mathrm{H} 2$ stated that foot placement accuracy would increase with longer exposure to ankle moment constraints. We thus expected a higher $\mathrm{R}^{2}$ at the end of the training as compared to the start of the training. H3 stated that there would be an after-effect when walking with normal shoes after the training sessions. We thus expected a higher $\mathrm{R}^{2}$ at the start of the 
185 after-effect condition as compared to the end of the baseline trial. $\mathrm{H} 4$ stated that over time the $\mathrm{R}^{2}$ during

186 the after-effect condition would decrease towards its baseline level. We thus expected the $\mathrm{R}^{2}$ at the end

187 of the after-effect condition to be lower than at the start. Moreover, we explored stride time, step width

188 and local divergence at the same time points. For all statistical tests, $p<0.05$ was treated as significant. 


\section{Results}

Nineteen healthy adults participated in this experiment. Four participants were excluded from further analysis due to measurement errors that affected the calculations. All data were normally distributed. On average participants took $529(\mathrm{SD}=16)$ steps in the 10 -minute baseline condition, $836(\mathrm{SD}=35)$ steps in the 15-minute training condition and $525(\mathrm{SD}=18)$ steps in the 10 -minute after-effect condition. See figures 2-5 for the observed gait changes during the three conditions.

\section{Baseline condition}

There were significant differences in $\mathrm{R}^{2}$ (figure 2), stride time (figure 3) and step width (figure 4) when comparing the end to the start of the baseline trial $(p<0.05)$. We found no significant change in gait stability (figure 5) over the baseline measurement $(p>0.05)$.

\section{Foot placement}

Walking with LesSchuh led to an immediate decrease in $\mathrm{R}^{2}$ (figure $2, p<0.05$ ), supporting H1. A significant increase in $\mathrm{R}^{2}$ over the training trial (supporting $\mathrm{H} 2, p<0.05$ ) was found. There was no significant difference between the end of the baseline trial and the start of the after-effect trial $(p>0.05)$, refuting $\mathrm{H} 3$ that there would be an after-effect following the training with LesSchuh. We had hypothesized that an after-effect, if present, would decrease over time (H3), but there was no significant difference in $\mathrm{R}^{2}$ between the start and the end of the after-effect trial $(p>0.05)$.

a.

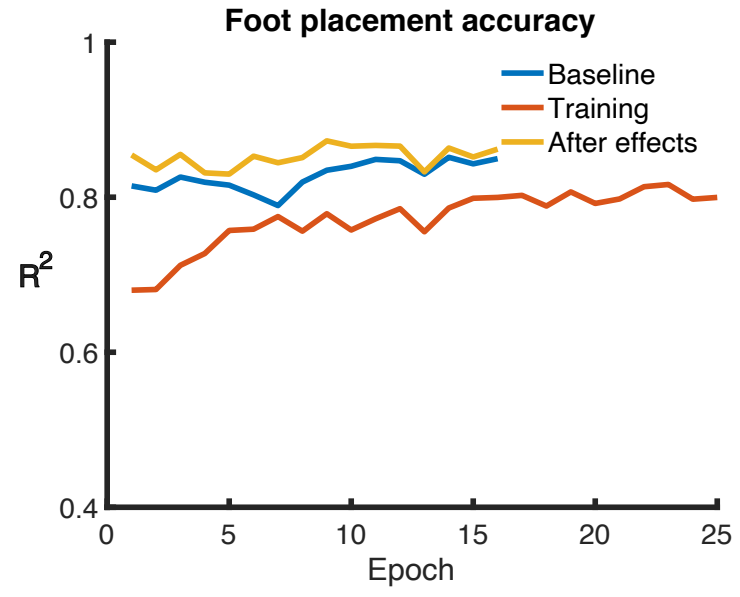

b.

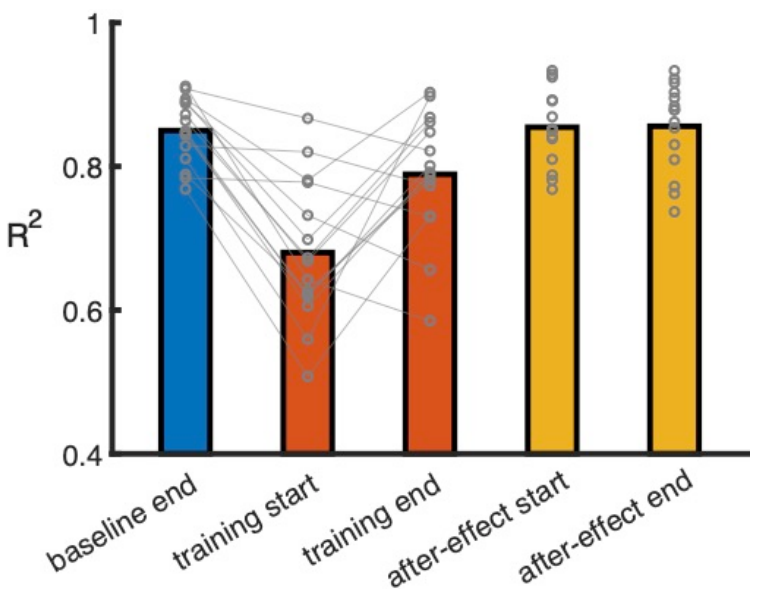

Figure 2. Foot placement accuracy (during the baseline, training and after-effect conditions). (a) Mean relative explained variance $\left(\mathrm{R}^{2}\right)$ of the foot placement prediction model across 30-strides episodes, (b) Mean $\mathrm{R}^{2}$ (and individual data points in gray) at the end of the baseline condition, the start and end of the training condition and at the start and end of the after-effect condition. For the significant effects, the individual data points have been connected. For illustrative purposes, in figure 2a, the data is depicted into epochs of 30 steps up to the number of epochs for which all participants had a full final epoch (i.e. including 30 steps).

\section{Stride time}

Exploratory analysis showed that stride time was significantly shorter at the start of the training trial as compared to the end of the baseline trials $(p<0.05)$. During the training, stride time increased again, resulting in a significant difference in stride time between the end and the start of the training $(p<0.05)$. At the start of the after-effect trial, stride time was still significantly shorter than at the end of the baseline 
bioRxiv preprint doi: https://doi.org/10.1101/2021.08.13.456273; this version posted August 15, 2021. The copyright holder for this preprint (which was not certified by peer review) is the author/funder, who has granted bioRxiv a license to display the preprint in perpetuity. It is made available under aCC-BY-NC-ND 4.0 International license.

trial $(p<0.05)$. This effect washed-out, as there was a significant increase in stride time from the start until the end of the after-effect condition $(p<0.05)$.

a.

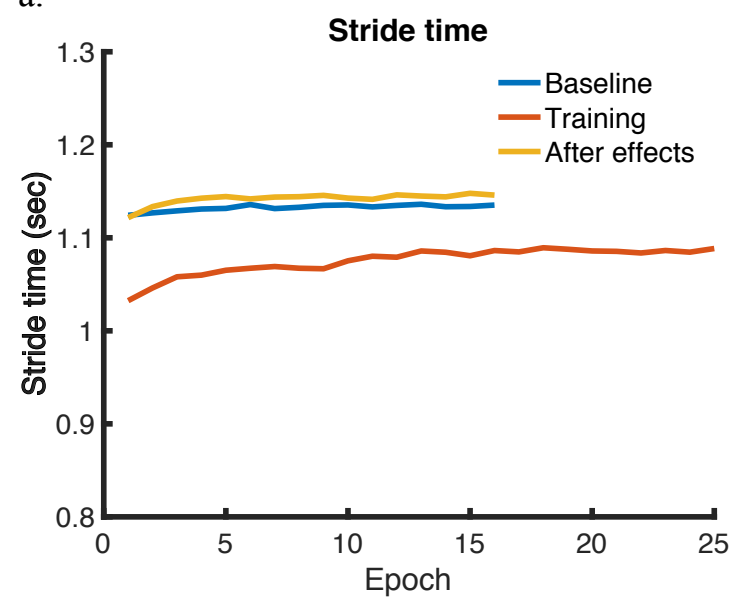

b.

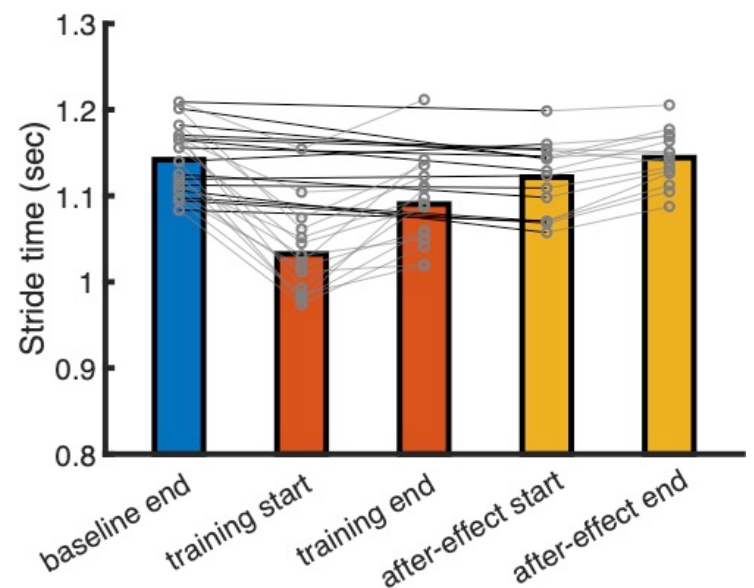

Figure 3. Stride times (during the baseline, training and after-effect conditions). (a) Mean stride time across stride episodes (b) Mean stride (and individual data points in gray) at the end of the baseline condition, the start and end of the training condition and at the start and end of the after-effect condition. For the significant effects, the individual data points have been connected. For illustrative purposes, in figure $3 \mathrm{a}$, the data is depicted into epochs of 30 steps up to the number of epochs for which all participants had a full final epoch (i.e. including 30 steps).

\section{Step width}

Step width increased compared to baseline when walking with LesSchuh at the start of the training $(p<0.05)$. There was no significant decrease in step width during the training $(p>0.05)$, nor a significant after-effect $(p>0.05)$. In line with this, there was no significant difference between the start and the end of the after-effect trial $(p>0.05)$.

a)

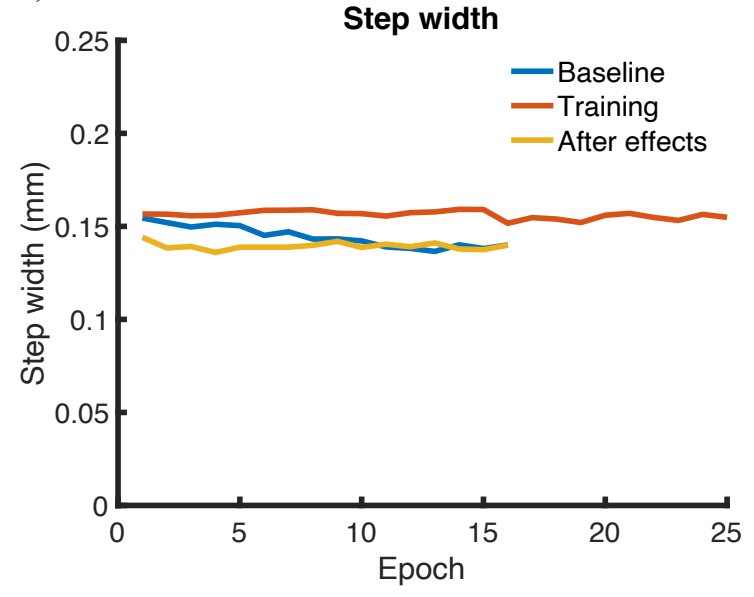

b)

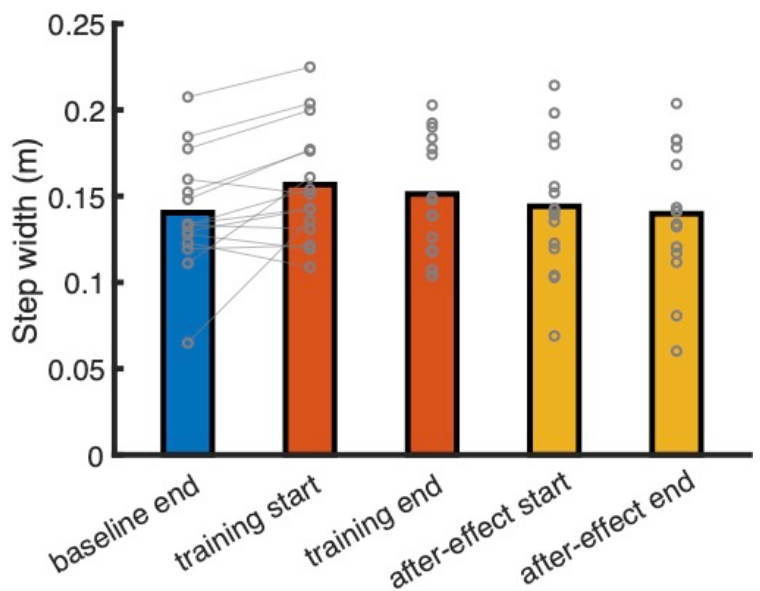

Figure 4. Step width (during the baseline, training and after-effect conditions). (a) Mean step width across stride episodes, (b) Mean step width (and individual data points in gray) at the end of the baseline condition, the start and end of the training condition and at the start and end of the after-effect condition. For the significant effect, the individual data points have been connected. For illustrative purposes, in figure $4 \mathrm{a}$, the data is depicted into epochs of 30 steps up to the number of epochs for which all participants had a full final epoch (i.e. including 30 steps 
bioRxiv preprint doi: https://doi.org/10.1101/2021.08.13.456273; this version posted August 15, 2021. The copyright holder for this preprint (which was not certified by peer review) is the author/funder, who has granted bioRxiv a license to display the preprint in perpetuity. It is made available under aCC-BY-NC-ND 4.0 International license.

\section{Gait stability}

The local divergence exponent (figure 5) significantly increased (i.e. local dynamic stability decreased) when walking with LesSchuh at the start of the training as compared to the end of the baseline trial $(p<0.05)$. There was no significant change during the training, nor was there an aftereffect $(p>0.05)$. In line with this, there was no change in stability between the start and the end of the after-effect condition $(p>0.05)$.

a)

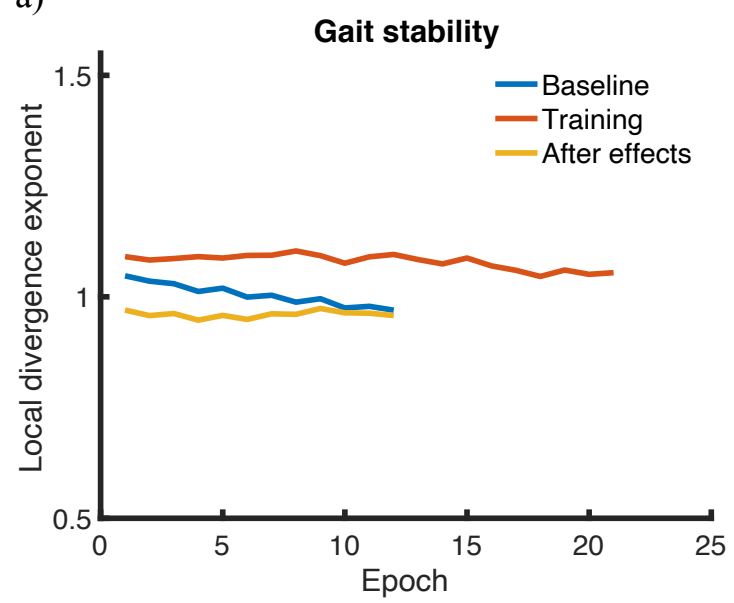

b)

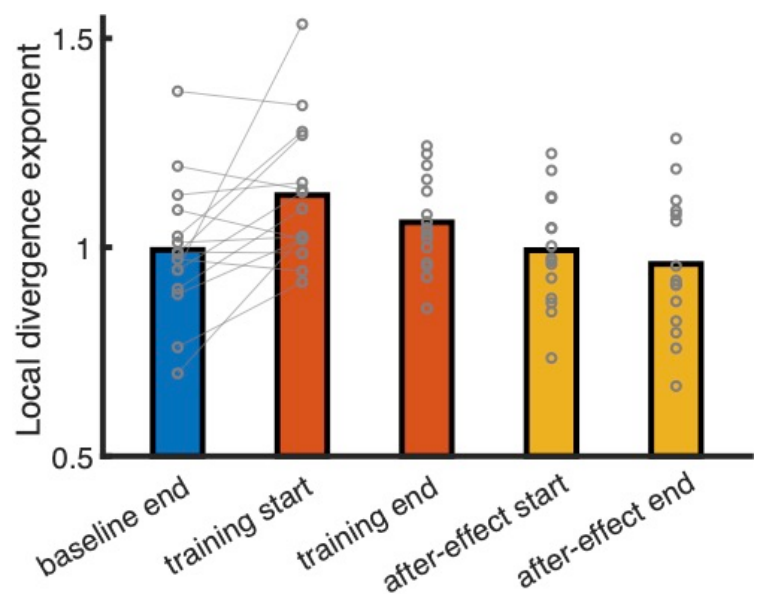

Figure 5. Gait stability (during the baseline, training and after-effect conditions. (a) Mean local divergence exponents across stride episodes, (b) Mean local divergence exponents (and individual data points in gray) at the end of the baseline condition, the start and end of the training condition and at the start and end of the after-effect condition. A lower local divergence exponent represents increased gait stability. For the significant effect, the individual data points have been connected. For illustrative purposes, in figure $5 \mathrm{a}$, the data is depicted into epochs of 30 steps up to the number of epochs for which all participants had a full final epoch (i.e. including 30 steps 


\section{Discussion}

The main purpose of this study was to investigate whether foot placement becomes more accurate after walking with shoes constraining ankle moments. We used ankle moment constraints as a foot placement perturbation to train foot placement accuracy. We verified that foot placement accuracy decreased while walking with shoes constraining ankle moments. We also observed increasing foot placement accuracy during longer exposure to the ankle moment constraining shoes. However, despite this adaptation, foot placement accuracy did not significantly increase as an after-effect upon returning to normal shoes. As an exploratory analysis, we assessed changes in stride time, step width and local dynamic stability alongside the effects on foot placement accuracy. We showed that walking with ankle moment constraints diminishes stability and coincided with compensatory adjustments in step width and stride time. We speculate that further improvement of foot placement accuracy may be achieved with a longer training period, and that the non-significant trend towards an after-effect reflects a training potential. Below we discuss the gait changes per condition.

\section{Gait changes during the baseline condition}

During the baseline condition, foot placement accuracy, stride time and step width changed significantly from the start until the end of the trial. Such changes reflect the need for habituation to treadmill walking. We did not use a familiarization period prior to the baseline condition. This may limit the interpretation of our results. However, previous research showed that 6-7 minutes (425 strides) of treadmill walking is sufficient to achieve stable gait parameters (Meyer et al., 2019). Since participants walked for 10 minutes in the baseline condition, one may reasonably assume the last 30 strides of these trials to provide a valid reference for further comparisons.

\section{Gait changes in the training condition}

In the training condition, participants walked while wearing LesSchuh (Figure 1). As expected from earlier results (van Leeuwen et al., 2020), foot placement accuracy decreased and step width and stride frequency increased. Increasing step width and stride frequency appears to be an appropriate strategy to maintain stability, in spite of reduced foot placement accuracy (Hak et al., 2013; Perry and Srinivasan, 2017). However, here local dynamic stability diminished when ankle moments were constrained, despite these compensatory mechanisms. Moreover, during unconstrained steady-state walking, step width and stride frequency seem to be largely determined by an energetic optimum (Delextrat et al., 2011; Donelan et al., 2001). Thus, increasing step width and frequency likely increases energy cost. Adopting a careful gait strategy (Wu et al., 2015) could be a temporary compensatory strategy that would disappear when foot placement accuracy is regained, and a more efficient step width (Donelan et al., 2001; Perry and Srinivasan, 2017) and stride time can be adopted.

Indeed, throughout the training condition, foot placement accuracy gradually improved towards the baseline level, suggesting a training potential. A higher foot placement accuracy is expected to allow a narrower step width while remaining equally stable (Perry and Srinivasan, 2017). However, although stride time increased again throughout the training, step width remained enlarged. Perhaps this is related to the fact that, at the end of the training condition, foot placement was still less accurate than during baseline walking. Possibly, foot placement accuracy will continue to improve with longer exposure to ankle moment constrained walking, before allowing a stable, energetically efficient gait pattern.

\section{Gait changes in the after-effect condition}

Similar to the after-effect of direct mechanical foot placement perturbations (Heitkamp et al., 2019), we expected more accurate foot placement in the after-effect condition. However, although foot placement accuracy appeared higher throughout the after-effect trial compared to baseline (Figure 2), this difference was not significant. Therefore, we cannot conclude that walking with LesSchuh leads to an after-effect. We did not prove that constraining ankle moments can lead to a training effect on foot placement accuracy during normal steady-state walking. Still, we argue that these results suggest a training potential of walking with constrained ankle moments. 
For every epoch, average foot placement accuracy was higher in the after-effect condition as compared to the baseline condition. Taken together with the increase in foot placement accuracy during training, we speculate that walking on LesSchuh for a longer duration, may induce a larger after-effect, potentially reaching statistical significance. Alternatively, it may not be the duration but the participant group which resulted in a statistically undiscernible after-effect. Young adults already have a relatively high foot placement accuracy (Arvin et al., 2018; Hurt et al., 2010; Wang and Srinivasan, 2014), which may limit their capacity for improvement. Therefore, a larger training effect could be expected in older adults (Arvin et al., 2018; Hurt et al., 2010) and patients with an impaired gait pattern (Dean and Kautz, 2015). Lastly, training while imposing additional constraints on other compensatory strategies, i.e. on average step width and stride frequency, may be more effective in enhancing foot placement accuracy. In any case, future studies should include a control experiment during which participants walk for the same duration, but while wearing normal shoes during all trials. This could clarify whether any "aftereffect" truly reflects a training potential, rather than treadmill familiarization.

\section{Conclusion}

Walking with ankle moment constraints perturbed foot placement control. This was reflected by a decrease in foot placement accuracy in relation to the center of mass kinematic state. With longer exposure to the ankle moment constraint, participants adapted, showing increased foot placement accuracy over time. When walking with normal shoes again, no significant after-effects were found. Nevertheless, there were indications of a training potential of walking with ankle moment constraints. It seems the interdependency of ankle moment and foot placement control provides an opportunity for

317 training interventions.

319 The authors are thankful for the participants and (technical) assistance during the experiment. We are 


\section{References}

Arvin, M., Hoozemans, M., Pijnappels, M., Duysens, J., Verschueren, S.M.P., Van Dieen, J., 2018. Where to step? Contributions of stance leg muscle spindle afference to planning of mediolateral foot placement for balance control in young and older adults. Frontiers in physiology 9, 1134.

Bruijn, S., Meijer, O., Beek, P., Van Dieën, J., 2013. Assessing the stability of human locomotion: a review of current measures. Journal of the Royal Society Interface 10, 20120999.

Bruijn, S.M., 2021. SjoerdBruijn/LocalDynamicStability: Latest release, including multiple nn (1.1). Zenodo. https://doi.org/10.5281/zenodo.4681213.

Bruijn, S.M., van Dieën, J.H., 2018. Control of human gait stability through foot placement. Journal of The Royal Society Interface 15, 20170816.

Bruijn, S.M.v.L., A.M., 2020. SjoerdBruijn/FootPlacement: Initial Release (Version V1.0). Zenodo http://doi.org/10.5281/zenodo.4292212.

De Leva, P., 1996. Adjustments to Zatsiorsky-Seluyanov's segment inertia parameters. Journal of biomechanics 29, 1223-1230.

Dean, J.C., Kautz, S.A., 2015. Foot placement control and gait instability among people with stroke. Journal of rehabilitation research and development 52, 577.

Delextrat, A., Matthew, D., Cohen, D.D., Brisswalter, J., 2011. Effect of stride frequency on the energy cost of walking in obese teenagers. Human movement science 30, 115-124.

Donelan, J.M., Kram, R., Arthur D, K., 2001. Mechanical and metabolic determinants of the preferred step width in human walking. Proceedings of the Royal Society of London. Series B: Biological Sciences 268, 1985-1992.

Fettrow, T., Reimann, H., Grenet, D., Thompson, E., Crenshaw, J., Higginson, J., Jeka, J., 2019. Interdependence of balance mechanisms during bipedal locomotion. bioRxiv, 658120 . Florence, C.S., Bergen, G., Atherly, A., Burns, E., Stevens, J., Drake, C., 2018. Medical costs of fatal and nonfatal falls in older adults. Journal of the American Geriatrics Society 66, 693698.

Hak, L., Houdijk, H., Beek, P.J., van Dieen, J.H., 2013. Steps to take to enhance gait stability: the effect of stride frequency, stride length, and walking speed on local dynamic stability and margins of stability. PLoS One 8, e82842.

Heitkamp, L.N., Stimpson, K.H., Dean, J.C., 2019. Application of a novel force-field to manipulate the relationship between pelvis motion and step width in human walking. IEEE Transactions on Neural Systems and Rehabilitation Engineering 27, 2051-2058. Hof, A.L., 2007. The equations of motion for a standing human reveal three mechanisms for balance. Journal of biomechanics 40, 451-457.

Hurt, C.P., Rosenblatt, N., Crenshaw, J.R., Grabiner, M.D., 2010. Variation in trunk kinematics influences variation in step width during treadmill walking by older and younger adults. Gait \& posture 31, 461-464.

Mahaki, M., Bruijn, S.M., Van Dieën, J.H., 2019. The effect of external lateral stabilization on the use of foot placement to control mediolateral stability in walking and running. PeerJ 7 , e7939.

Mehdizadeh, S., 2019. A robust method to estimate the largest lyapunov exponent of noisy signals: a revision to the rosenstein's algorithm. Journal of biomechanics 85, 84-91. Meyer, C., Killeen, T., Easthope, C.S., Curt, A., Bolliger, M., Linnebank, M., Zörner, B., Filli, L., 2019. Familiarization with treadmill walking: How much is enough? Scientific reports $9,1-10$. 
Perry, J.A., Srinivasan, M., 2017. Walking with wider steps changes foot placement control, increases kinematic variability and does not improve linear stability. Royal Society open science 4, 160627.

Polinder, S., Haagsma, J., Panneman, M., Scholten, A., Brugmans, M., Van Beeck, E., 2016. The economic burden of injury: Health care and productivity costs of injuries in the Netherlands. Accident analysis \& prevention 93, 92-100. Rebula, J.R., Ojeda, L.V., Adamczyk, P.G., Kuo, A.D., 2017. The stabilizing properties of foot yaw in human walking. Journal of biomechanics 53, 1-8. Reimann, H., Fettrow, T., Jeka, J.J., 2018. Strategies for the Control of Balance During 380 Locomotion. Kinesiology Review 7, 18-25.

381 Reimold, N.K., Knapp, H.A., Henderson, R.E., Wilson, L., Chesnutt, A.N., Dean, J.C., 2019. 382 Altered active control of step width in response to mediolateral leg perturbations while 383 walking. bioRxiv.

384 Roerdink, M., Lamoth, C.J., Beek, P.J., 2008. Online gait event detection using a large force platform embedded in a treadmill. Journal of biomechanics 41, 2628-2632.

386 Rosenstein, M.T., Collins, J.J., De Luca, C.J., 1993. A practical method for calculating largest Lyapunov exponents from small data sets. Physica D: Nonlinear Phenomena 65, 117-134.

388 Rubenstein, L.Z., 2006. Falls in older people: epidemiology, risk factors and strategies for 389 prevention. Age and ageing 35, ii37-ii41.

390 Rubenstein, L.Z., Josephson, K.R., 2006. Falls and their prevention in elderly people: what 391 does the evidence show? Medical Clinics 90, 807-824.

392 van Leeuwen, A.M., van Dieen, J.H., Daffertshofer, A., Bruijn, S.M., 2020. Active foot 393 placement control ensures stable gait: Effect of constraints on foot placement and ankle 394 moments. Plos one 15, e0242215.

395 Vandervoort, A.A., 2002. Aging of the human neuromuscular system. Muscle \& Nerve: 396 Official Journal of the American Association of Electrodiagnostic Medicine 25, 17-25. 397 Wang, Y., Srinivasan, M., 2014. Stepping in the direction of the fall: the next foot placement 398 can be predicted from current upper body state in steady-state walking. Biology letters 10. 399 Wu, M., Matsubara, J.H., Gordon, K.E., 2015. General and specific strategies used to 400 facilitate locomotor maneuvers. PloS one 10.

401 Zatsiorsky, V., 1990. Methods of determing mass-inertial characteristics of human body 402 segments. Contemporasy Problems of Biomechnics.

403 Zhang, Y., Smeets, J.B., Brenner, E., Verschueren, S., Duysens, J., 2020. Fast responses to 404 stepping-target displacements when walking. The Journal of Physiology. 